\title{
A comparison of ambulatory perioperative times in hospitals and freestanding centers
}

\author{
Brionna Hair, M.P.H. ${ }^{a}{ }^{*}$, Peter Hussey, Ph.D. ${ }^{b}$, and Barbara Wynn, M.A. ${ }^{b}$ \\ aDepartment of Epidemiology, University of North Carolina at Chapel Hill, CB \#7435, 2101 \\ McGavran-Greenberg Hall, Chapel Hill, NC 27599-7435, USA

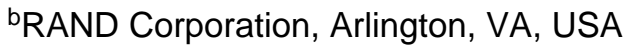

\section{Abstract}

BACKGROUND-The volume of surgical procedures performed in ambulatory surgical centers has increased rapidly.

\begin{abstract}
METHODS-Ambulatory surgical visits of Medicare beneficiaries were compared for hospitalbased and freestanding ambulatory surgical centers (ASCs). The main outcomes were time in surgery, time in operating room, time in postoperative care, and total perioperative time.
\end{abstract}

\begin{abstract}
RESULTS-The mean total perioperative time for all procedures examined was $39 \%$ shorter in freestanding ASCs then in hospital-based ASCs (83 vs $135 \mathrm{~min} ; P<.01$ ); surgery time was $37 \%$ shorter (19 vs $30 \mathrm{~min} ; P<.01$ ), operating room time was $37 \%$ shorter ( 34 vs $54 \mathrm{~min} ; P<.01$ ), and postoperative time was $35 \%$ shorter (48 vs $74 \mathrm{~min} ; P<.01$ ).
\end{abstract}

CONCLUSIONS-Perioperative times were significantly shorter in freestanding ASCs than in hospital-based ASCs. It is unclear how much of the difference was the result of efficiency versus patient selection.

\section{Keywords}

Ambulatory surgery; Ambulatory surgery center; Hospital outpatient department; Anesthesia use; Surgical time

The past 3 decades have seen substantial growth in ambulatory surgery performed in freestanding ambulatory surgery centers (ASCs) in the United States. ${ }^{1,2}$ In 2006, approximately $43 \%$ of 34.7 million ambulatory surgery visits took place in freestanding ASCs. ${ }^{2}$ This growth has been attributed in part to the degree of control that freestanding ASCs afford surgeons over their professional lives through authority over staffing, surgical equipment, and scheduling. ${ }^{1,3}$ Freestanding ASCs may function as "focused factories," allowing surgeons to achieve greater productivity relative to practicing in hospital outpatient departments. ${ }^{1}$ Some differences in productivity also may be owing to physicians with ownership stakes in freestanding ASCs systematically referring lower-risk patients or more profitable procedures to freestanding ASCs instead of hospital outpatient departments. ${ }^{4,5}$

Medicare payment policy implicitly recognizes that freestanding ASCs perform surgical procedures at a lower cost than hospital outpatient departments. Since 2008, freestanding

(C) 2012 Elsevier Inc. All rights reserved.

*Corresponding author. Tel.: +1-202-669-3152; fax: +1-919-966-4916. bhair@email.unc.edu.

Work originated from the RAND Corporation.

Peter Hussey and Barbara Wynn are employees of RAND Corporation; Brionna Hair reports no conflicts of interest. 
ASCs have been reimbursed at a fixed percentage (61\% in 2011) of the hospital rate for equivalent services. However, the payment differential is calculated on the basis of budget neutrality with previous payment systems and not on the basis of estimates of the cost of providing services in each setting.

Previous studies have found that for selected procedures freestanding ASCs provide care with equivalent safety and shorter surgical times relative to hospital outpatient departments. ${ }^{6-9}$ However, no studies have compared surgical times for a comprehensive set of ambulatory procedures performed in both hospitals and freestanding ASCs. The objective of this study was to compare surgical times for ambulatory procedures commonly performed in both hospitals and freestanding ASCs. A secondary objective was to compare anesthesia use by facility type.

\section{Methods}

The 2006 National Survey of Ambulatory Surgery (NSAS) public use data file, a survey of ambulatory procedures performed in hospitals and freestanding ASCs in the United States, was used for all analyses. A detailed description of the design and methodology used by the survey has been described previously. ${ }^{10}$ Briefly, the NSAS uses a multistage probability design to sample hospitals and freestanding ASCs. ${ }^{11}$ An ASC was considered hospital-based if it was a facility that was licensed as a hospital and offered ambulatory surgery. The surgery could have occurred in a general operating room, in a room or facility dedicated to ambulatory surgery, or in a room dedicated to specialized procedures. ${ }^{10}$ A freestanding ASC was considered eligible for NSAS if it was either regulated by one of the states in the U.S. or was certified for participation in Medicare. In the 2006 survey, 142 of 189 eligible hospitals and 295 of 397 eligible freestanding ASCs responded. For each sampled facility, systematic random sampling was used to select a sample of ambulatory surgery visits. Data were abstracted for selected visits using a medical abstract form.

All analyses in this study were restricted to visits in which a single surgical procedure was performed, in which Medicare was the principle source of payment, and in which the patient routinely was discharged home. We compared hospital-based ASCs and freestanding ASCs on procedures in the following anatomic systems: nervous system (International Classification of Diseases, 9th revision, Clinical Modification [ICD-9-CM] procedure codes 01-05), eye system (ICD-9-CM procedure codes 08-16), cardiovascular system (ICD-9-CM procedure codes 35-39), digestive system (ICD-9-CM procedure codes 42-54),

musculoskeletal system (ICD-9-CM procedure codes 76-84), integumentary system (ICD-9CM procedure codes $85-86$ ), and miscellaneous diagnostic and therapeutic procedures (ICD-9-CM procedure codes 87-99); and by the following selected procedures: release of carpal tunnel (ICD-9-CM procedure code 04.44), extraction of lens (ICD-9-CM procedure codes 13.1-13.6), other endoscopy of the small intestine (ICD-9-CM procedure code 45.13), endoscopic polypectomy of the large intestine (ICD-9-CM procedure code 45.42), closed (endoscopic) biopsy of the large intestine (ICD-9-CM procedure code 45.25), other local excision or destruction of lesion or tissue of skin and subcutaneous tissue (ICD-9-CM procedure code 86.3), upper-gastrointestinal endoscopy, biopsy (ICD-9-CM procedure codes 45.16 and 44.14), and diagnostic colonoscopy (ICD-9-CM procedure codes 45.22, 45.23, and 46.85 ). Only procedures that had at least 25 unweighted observations for both facility types were analyzed.

Our main outcome of interest was surgical time. We assessed 4 time periods in our analyses: total time, the time between when the patient entered the operating room and left postoperative care; surgery time, the time between when surgery began and when surgery 
ended; operating room time, the length of time spent in the operating room; and postoperative time, the length of time spent in postoperative care.

Descriptive statistics were used to compare the hospital-based and freestanding ASC visits by age distribution, sex, number of diagnoses reported at the time of visit, number of symptoms occurring during the procedure, and anesthesia use overall and for select procedures. The $t$ test was used to test differences in mean surgical times by facility type, accounting for the survey design. SAS 9.2 (SAS Institute, Cary, NC) and SUDAAN 10.0 (RTI, Research Triangle Park, NC) were used to perform statistical analyses.

\section{Results}

Our sample included a total of 5,510,493 visits listing a single procedure performed on routinely discharged patients with Medicare as the principal payer. Table 1 presents a comparison of selected characteristics of the visits by facility type. The age and gender distribution of the visits were not significantly different between the facility types. Hospitalbased ASCs reported a higher number of diagnoses per patient than freestanding ASCs (2.4 vs 1.7 diagnoses; $P<.01$ ). There were no marked differences in the reporting of symptoms related to the surgery. The most common symptoms reported were hypertension, nausea, and hypotension (data not shown).

Freestanding ASCs were more likely to report using intravenous (IV) sedation during patient visits ( $49 \%$ vs $41 \%$ ) than hospital-based centers overall, but were less likely to report use of general anesthesia (15\% vs $21 \%$ ) (Table 2). The use of anesthesia between the facility types also varied for upper-gastrointestinal endoscopy and diagnostic colonoscopy, procedures for which moderate sedation is indicated based on Appendix G of the Current Procedural Terminology code book. For upper-gastrointestinal endoscopy, freestanding ASCs reported more frequent use of IV sedation and monitored anesthesia care; for diagnostic colonoscopy, freestanding ASCs reported more frequent use of monitored anesthesia care and general anesthesia, but similar uses of IV sedation.

Table 3 presents comparisons of surgical times for procedures with at least 25 unweighted observations by facility type. The mean total time for all procedures was $39 \%$ shorter in freestanding ASCs than hospital-based ASCs ( 83 vs 135 min; $P<.01$ ). The mean total time was shorter in freestanding ASCs for most categories of procedures. The exceptions were procedures for the eye, cardiovascular system, and local excisions, for which there was no statistically significant difference in the mean total time between settings. The mean time was shorter in freestanding ASCs than hospital-based ASCs across 3 subperiods of time: compared with hospitals, ASC surgery time was $37 \%$ shorter (19 vs $30 \mathrm{~min} ; P<.01$ ), operating room time was $37 \%$ shorter (34 vs $54 \mathrm{~min} ; P<.01$ ), and postoperative time was $35 \%$ shorter (48 vs $74 \mathrm{~min} ; P<.01$ ).

\section{Comments}

This study found that in the Medicare population with visits resulting in discharge to home, freestanding ASCs perform surgeries in less time than hospital-based ASCs overall and for procedures on various anatomic systems. The difference in average surgical times was approximately equal to the difference in 2011 Medicare payment amounts per relative value unit (freestanding ASCs at 56\% of hospitals). Our results corroborate the notion that freestanding ASCs tend to be more efficient than hospital-based surgery centers. Trentman et $\mathrm{al}^{6}$ examined breast surgeries in a freestanding ASC and in the hospital setting and reported that in their freestanding ASC the total time in the facility was 69 minutes shorter than when the same procedures were performed in a hospital setting, although surgeries in 
the hospital consisted of both inpatient and outpatient surgeries. Most of the reduction in time was during the preoperative phase, which differed from our results that indicated freestanding ASCs were shorter in actual surgery time, time in the operating room, and in postoperative time; data were not available on preoperation surgical time in the NSAS. Trent-man et $\mathrm{al}^{6}$ did find that freestanding ASC patients spent less time in the postanesthesia care unit than hospital patients (112 vs $121 \mathrm{~min} ; P=.16$ ), which was not as substantial a difference as the time spent in postoperative care that our study found (74 vs $48 \mathrm{~min} ; P<$. $01)$.

Our analyses also suggest that there are differences in practice between ASCs and hospitalbased ambulatory surgery with regards to the use of anesthesia during surgery. Freestanding ASCs were more likely to use IV sedation and monitored anesthesia care for uppergastrointestinal biopsy, and were more likely to use IV sedation overall. The Trent-man et $\mathrm{al}^{6}$ study found that use of anesthesia was similar in the freestanding ASCs and the hospital, although they reported that during surgery higher doses of fentanyl and ketorolac were used. The differences in anesthesia use in freestanding ASCs partially may explain the shorter time spent in postoperative care found in our study because there was a shorter recovery time associated with the use of moderate sedation than with the use of general anesthesia. ${ }^{12,13}$

Our study had several important limitations. The NSAS definition of ambulatory surgery results in the grouping together of a variety of surgical styles among the hospital-based ASCs. We were unable to distinguish between ambulatory surgeries that occurred in the main operating room of a hospital, which may have been more likely to be influenced by the operational logistics associated with hospital outpatient surgery, and surgeries that occurred in a hospital-owned facility located in a site distinct from the hospital, which may be more likely to be run in a fashion similar to freestanding ASCs. This mixing of surgical styles among the hospital-based ASCs may have diluted the estimated differences in surgical times between facility types.

There were little detailed patient-level data available. Although the age and gender distribution of patient visits served by the 2 facility types were similar, our analyses were unable to account for possible differences in the case mix between the 2 facility types, although the number of diagnoses, which were somewhat higher in the hospital-based ambulatory surgery visits, may be used as a proxy for comorbidity. Evidence suggests that freestanding ASCs see patients with less comorbidity and complexity than hospitals. ${ }^{7}, 14 \mathrm{We}$ also were unable to analyze facility-level factors, such as patient volume, facility ownership, teaching status of the hospital, and characteristics of the surgeons performing procedures, which may have affected the comparability of surgeries performed in this study. Also, because of small unweighted sample sizes, we were unable to examine surgical times for more specific procedures, which would have facilitated more apt comparisons between freestanding and hospital-based ASCs.

Despite the limitations, our study adds to the evidence that ambulatory surgeries performed in freestanding centers are more efficient than surgery performed in hospital-based centers. It is important to understand what drives the higher efficiency of freestanding ASCs so the successful elements of their practice can be adopted by other facility types, as appropriate. As the population of the United States ages and more citizens become eligible for Medicare, more efficient, high-quality care will be essential. Future research should address how to improve efficiency in hospital-based ambulatory surgery centers while containing costs. 


\section{Acknowledgments}

This project was conducted under a contract with the US Department of Health and Human Services Office of the Assistant Secretary for Planning and Evaluation. The funder provided input on the study design and commented on the study findings.

\section{References}

1. Casalino LP, Devers KJ, Brewster LR. Focused factories? Physician-owned specialty facilities. Health Aff (Millwood). 2003; 22:56-67. [PubMed: 14649432]

2. Cullen KA, Hall MJ, Golosinskiy A. Ambulatory surgery in the United States, 2006. Revised. Natl Health Stat Rep. 2009; 11:1-25.

3. Hollingsworth JM, Ye Z, Strope SA, et al. Physician-ownership of ambulatory surgery centers linked to higher volume of surgeries. Health Aff (Millwood). 2010; 29:683-689. [PubMed: 20368599]

4. Gabel JR, Fahlman C, Kang R, et al. Where do I send thee? Does physician-ownership affect referral patterns to ambulatory surgery centers? Health Aff (Millwood). 2008; 27:w165-w174. [PubMed: 18349040]

5. Plotzke MR, Courtemanche C. Does procedure profitability impact whether an outpatient surgery is performed at an ambulatory surgery center or hospital? Health Econ. 2011; 20:817-830. [PubMed: 20669335]

6. Trentman TL, Mueller JT, Gray RJ, et al. Outpatient surgery performed in an ambulatory surgery center versus a hospital: comparison of perioperative time intervals. Am J Surg. 2010; 200:64-67. [PubMed: 20637337]

7. Paquette IM, Smink D, Finlayson SR. Outpatient cholecystectomy at hospitals versus freestanding ambulatory surgical centers. J Am Coll Surg. 2008; 206:301-305. [PubMed: 18222383]

8. Grisel J, Arjmand E. Comparing quality at an ambulatory surgery center and a hospital-based facility: preliminary findings. Otolaryngol Head Neck Surg. 2009; 141:701-709. [PubMed: 19932841]

9. Fleisher LA, Pasternak LR, Herbert R, et al. Inpatient hospital admission and death after outpatient surgery in elderly patients: importance of patient and system characteristics and location of care. Arch Surg. 2004; 139:67-72. [PubMed: 14718279]

10. McLemore T, Lawrence L. Plan and operation of the national survey of ambulatory surgery. Vital Health Stat. 1997; 37:I-IV. 1-124.

11. National Center for Health Statistics. Revised Public Use Data File Documentation. Hyattsville, MD: 2009. National Survey of Ambulatory Surgery. 2006. Available at: ftp://ftp.cdc.gov/pub/ health_statistics/nchs/Dataset_Documentation/NSAS/NSAS2006rev.pdf [Accessed: June 2010]

12. Fedok FG, Ferraro RE, Kingsley CP, et al. Operative times, postanesthesia recovery times, and complications during sinonasal surgery using general anesthesia and local anesthesia with sedation. Otolaryngol Head Neck Surg. 2000; 122:560-566. [PubMed: 10740178]

13. Snyder SK, Roberson CR, Cummings CC, et al. Local anesthesia with monitored anesthesia care vs general anesthesia in thyroidectomy: a randomized study. Arch Surg. 2006; 141:167-173. [PubMed: 16490894]

14. Winter A. Comparing the mix of patients in various outpatient surgery settings. Health Aff (Millwood). 2003; 22:68-75. [PubMed: 14649433] 
Table 1

Characteristics of patient visits by facility type

\begin{tabular}{|c|c|c|c|c|}
\hline & Total & Hospital & FASC & $P$ value \\
\hline Total $^{*}$ & $5,510,493$ & $3,108,896$ & $2,401,597$ & \\
\hline \multicolumn{5}{|l|}{ Age } \\
\hline Mean, y (SE) & $71.0(.4)$ & $70.6(.4)$ & $71.4(.8)$ & .42 \\
\hline$<15 \mathrm{y}, \mathrm{n}(\%)$ & $17,018(.3)$ & $8,363(.3)$ & $8,655(.4)$ & \\
\hline $15-44 \mathrm{y}, \mathrm{n}(\%)$ & $166,528(3.0)$ & $108,699(3.5)$ & $57,829(2.4)$ & \\
\hline $45-64$ y, n (\%) & $698,755(12.7)$ & $435,878(14.0)$ & $262,877(10.9)$ & \\
\hline $65-74$ y, n (\%) & $2,430,491(44.1)$ & $1,312,266(42.2)$ & $1,118,225$ (46.6) & \\
\hline$\geq 75 \mathrm{y}, \mathrm{n}(\%)$ & $2,197,701(39.9)$ & $1,243,690(40.0)$ & $954,011(39.7)$ & \\
\hline Male, n (\%) & $2,444,348(44.4)$ & $1,407,478(45.3)$ & $1,036,870(43.2)$ & .23 \\
\hline \multicolumn{5}{|l|}{ Diagnoses, $\mathrm{n}$} \\
\hline Mean (SE) & $2.1(.1)$ & $2.4(.2)$ & $1.7(.1)$ & $<.001$ \\
\hline 1 & $2,729,429(49.5)$ & $1,374,572(44.2)$ & $1,354,857(56.4)$ & \\
\hline 2 & $1,270,468(23.1)$ & $660,401(21.2)$ & $610,067(25.4)$ & \\
\hline 3 & $687,929(12.5)$ & $381,413(12.3)$ & $306,516(12.8)$ & \\
\hline 4 & $294,840(5.4)$ & $207,240(6.7)$ & $87,600(3.6)$ & \\
\hline 5 & $200,521(3.6)$ & $173,810(5.6)$ & $26,711(1.1)$ & \\
\hline 6 & $155,797(2.8)$ & $146,049(4.7)$ & $9,748(.4)$ & \\
\hline 7 & $171,509(3.1)$ & $165,411(5.3)$ & $6,098(.3)$ & \\
\hline \multicolumn{5}{|l|}{ Symptoms, n } \\
\hline Mean (SE) & $.04(.01)$ & $.04(.01)$ & $.04(.01)$ & .99 \\
\hline 0 & $5,293,377(96.1)$ & $2,986,316(96.1)$ & $2,307,061(96.1)$ & \\
\hline 1 & 204,865 (3.7) & $115,702(3.7)$ & $89,163(3.7)$ & \\
\hline 2 & $10,022(.2)$ & $5,830(.2)$ & $4,192(.2)$ & \\
\hline 3 & $2,229(.0)$ & $1,048(.0)$ & $1,181(.0)$ & \\
\hline
\end{tabular}

FASC $=$ freestanding ambulatory surgery center; $\mathrm{SE}=$ standard error.

Total number of visits, based on weighted frequencies, for Medicare beneficiaries who underwent one procedure and were discharged home. 
Table 2

Anesthesia use by facility type, overall, and for select procedures

\begin{tabular}{|c|c|c|c|}
\hline & Total & Hospital & FASC \\
\hline Total visits ${ }^{*}, \mathrm{n}^{\dagger}$ & $5,510,493$ & $3,108,896$ & $2,401,597$ \\
\hline Topical, n (\%) & $1,020,561(19)$ & $530,006(17)$ & $490,555(20)$ \\
\hline IV sedation, $\mathrm{n}(\%)$ & $2,450,245(44)$ & $1,273,752(41)$ & $1,176,493(49)$ \\
\hline Monitored anesthesia care, $\mathrm{n}(\%)$ & $1,275,314(23)$ & $691,043(22)$ & $584,271(24)$ \\
\hline Regional epidural, n (\%) & $28,487(1)$ & $11,458(0)$ & $17,029(1)$ \\
\hline Regional spinal, n (\%) & $47,918(1)$ & $46,044(1)$ & $1,874(0)$ \\
\hline Regional retrobulbar block, $\mathrm{n}(\%)$ & $39,228(1)$ & $14,473(0)$ & $24,755(1)$ \\
\hline Regional peribulbar block, n (\%) & $7,294(0)$ & $2,395(0)$ & $4,899(0)$ \\
\hline Regional block, n (\%) & $106,509(2)$ & $51,483(2)$ & $55,026(2)$ \\
\hline General, n (\%) & $1,002,930(18)$ & $650,095(21)$ & $352,835(15)$ \\
\hline Other, n $(\%)$ & $114,045(2)$ & $59,932(2)$ & $54,113(2)$ \\
\hline None specified, n (\%) & $389,548(7)$ & $258,727(8)$ & $130,821(5)$ \\
\hline \multicolumn{4}{|l|}{ Upper GI endoscopy biopsy } \\
\hline Total, $\mathrm{n}^{t}$ & 382,955 & 210,817 & 172,138 \\
\hline Topical, n (\%) & $32,444(8)$ & $18,570(9)$ & $13,874(8)$ \\
\hline IV sedation, $\mathrm{n}(\%)$ & $281,212(73)$ & $147,136(70)$ & $134,076(78)$ \\
\hline Monitored anesthesia care, $\mathrm{n}(\%)$ & $97,790(26)$ & $40,318(19)$ & $57,472(33)$ \\
\hline General, n (\%) & $23,575(6)$ & $17,520(8)$ & $6,055(4)$ \\
\hline \multicolumn{4}{|l|}{ Diagnostic colonoscopy } \\
\hline Total, $\mathrm{n}^{\xi}$ & $1,121,017$ & 446,154 & 674,863 \\
\hline Topical, n (\%) & $57,947(5)$ & $31,012(7)$ & $26,935(4)$ \\
\hline IV sedation, $\mathrm{n}(\%)$ & $786,228(70)$ & $312,267(70)$ & $473,961(70)$ \\
\hline Monitored anesthesia care, $\mathrm{n}(\%)$ & $273,938(24)$ & $893,44(20)$ & $184,594(27)$ \\
\hline General, n (\%) & $106,243(9)$ & $15,344(3)$ & $90,899(13)$ \\
\hline
\end{tabular}

FASC $=$ freestanding ambulatory surgery center.

*

Based on weighted frequencies.

${ }^{\dagger}$ Total number of surgical visits for Medicare beneficiaries who underwent one procedure and were discharged home.

${ }^{*}$ Total number of visits during which an upper-gastrointestinal endoscopy was performed.

$\mathcal{S}_{\text {Total number of visits during which a diagnostic colonoscopy was performed. }}$

Percentages total more than $100 \%$ when more than one type of anesthesia was used per visit. 


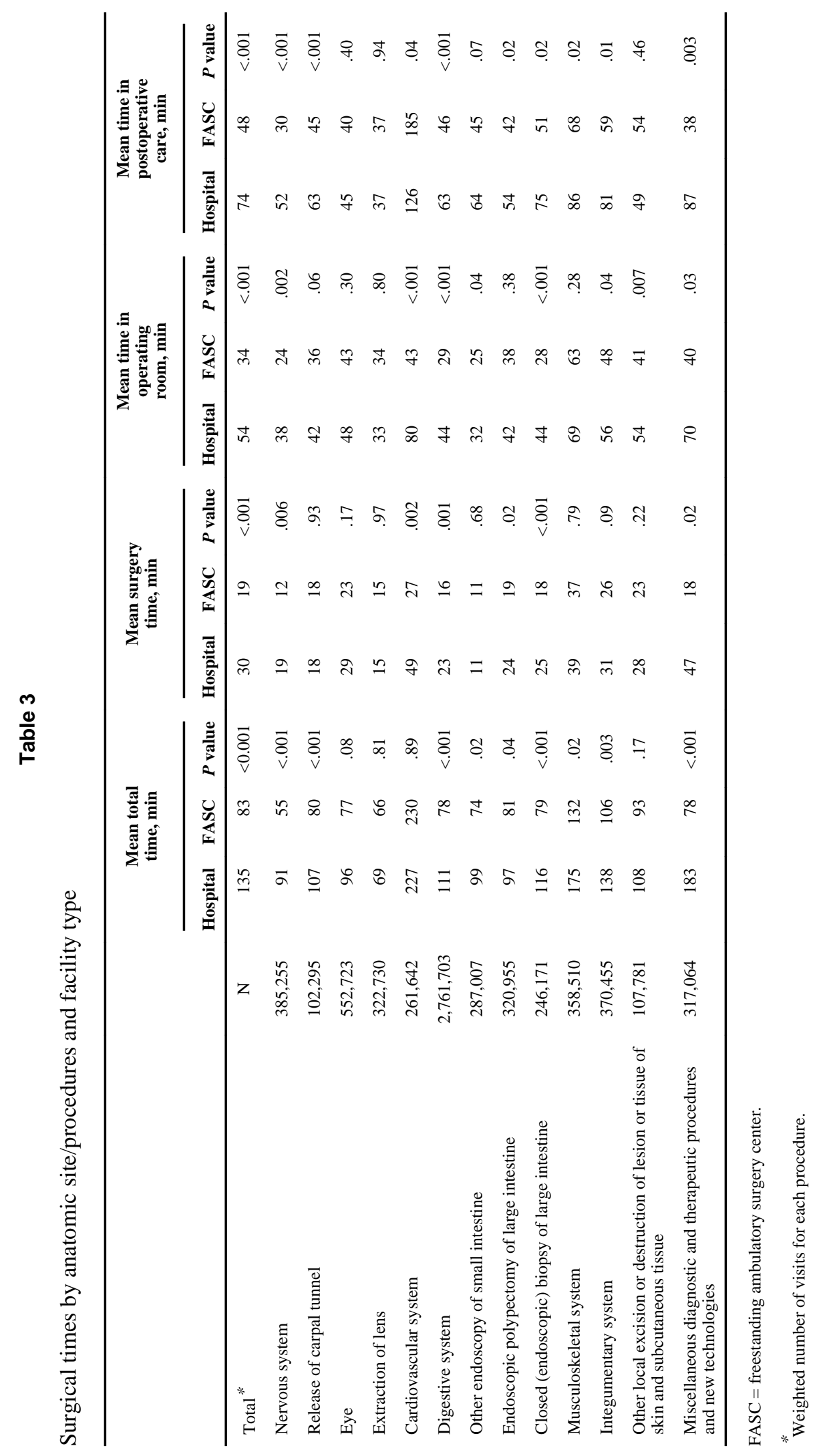

